\title{
Learning and encoding higher order rules in neural networks
}

\author{
DANIEL S. LEVINE \\ University of Texas, Arlington, Texas
}

\begin{abstract}
Some researchers state that whereas neural networks are fine for pattern recognition and categorization, complex rule formation requires a separate "symbolic" level. However, the human brain is a connectionist system and, however imperfectly, does complex reasoning and inference. Familiar modeling principles (e.g., Hebbian or associative learning, lateral inhibition, opponent processing, neuromodulation) could recur, in different combinations, in architectures that can learn diverse rules. These rules include, for example, "go to the most novel object," "alternate between two given objects," and "touch three given objects, without repeats, in any order." Frontal lobe damage interferes with learning all three of those rules. Hence, network models of rule learning and encoding should include a module analogous to the prefrontal cortex. They should also include modules analogous to the hippocampus for episode setting and analogous to the amygdala for emotional evaluation.
\end{abstract}

The growth of neural network research since the mid1980s has led to questions about the strengths and limitations of the neural network (connectionist) approach to modeling cognition. Some cognitive scientists (e.g., Fodor \& Pylyshyn, 1988) have even argued that the connectionist approach is inherently unsuitable for modeling some kinds of high-order cognitive processing. Specifically, Fodor, Pylyshyn, and others believe connectionist approaches are good for modeling pattern recognition and classification, but not for modeling those processes involving learning semantic relationships and rules. This has led to the popularity of hybrid models that combine neural networks with symbolic modules, both performing the type of operations they are supposed to be best suited for (see Bremner \& Gotts, 1995, and references therein).

Hybrid models, and pure symbolic models, certainly have wide utility in artificial intelligence applications. However, the human brain is a connectionist system, since it is composed of neurons and synapses with continuously varying electrical and chemical activities. We are able to perform semantic reasoning, learn inference, and do arithmetic, although not necessarily in an optimal manner (see, e.g., Anderson, Spoehr, \& Bennett, 1994). Hence, our brains are an existence proof that neural networks can perform high-order rule learning and encoding. A major goal of my current research, which is outlined in this article, is to see how they do so. This is a process likely to involve a large number of brain regions, but three regions seem to stand out as particularly important: the frontal lobes, hippocampus, and amygdala.

The question sometimes arises as to what is the appropriate level for modeling high-level cognitive processes. The neural network models discussed in this ar-

Correspondence should be addressed to D. S. Levine, Department of Mathematics, University of Texas at Arlington, 411 S. Nedderman Drive, Arlington, TX 76019-0408 (e-mail: b344dsl@utarlg.uta.edu). ticle are at the level of combining nodes, which typically represent concepts, sensory data, elementary movements, and so on, into functional architectures that can perform cognitive tasks. However, to complete the picture of how the brain might perform these tasks, we need to know what regions correspond to these nodes or functional units, and how neurons combine into nodes. While many neural net models at the higher level are based on good guesses about these nodes (subregions, cortical columns, etc.), there is concurrent research going on about how these nodes arise from networks of neurons or dendritic sites (see, e.g., Pribram, 1994).

A discussion of the issues about "neural networks versus traditional artificial intelligence" appears in Aparicio and Levine (1994). These issues will not be discussed in detail here, since I focus here on possible neural net mechanisms for rule formation. One of our main points is that critics such as Fodor and Pylyshyn based their anti-connectionist conclusions on a very small sample of the connectionist modeling literature. What they meant by a "connectionist system" was a type of neural network known as the back propagation network, or multilayer perceptron (see Werbos, 1974, or Rumelhart, Hinton, \& Williams, 1986). The capabilities of neural networks in general (see Levine, 1991, for a partial survey) are far greater than those of any one type of network, such as back propagation, adaptive resonance (Carpenter, Grossberg, \& Reynolds, 1991), Hopfield net (Hopfield \& Tank, 1986), or Kohonen net (Kohonen, 1988).

Perhaps more important, the neural network field is rapidly expanding, so it is important to distinguish potential capabilities of future neural networks from known capabilities of past and present ones. Typical networks designed for simulation of cognitive or behavioral data are themselves composed of smaller networks designed to do particular subtasks. If the network designed to simulate one set of psychological data is not capable of sim- 
ulating some other related but more complex data set, it may be possible to add more connections and subnetworks to the original network so that it can also simulate the other data set. If a researcher designs a network that can simulate behavior $X$, and someone criticizes the network because it cannot yet also simulate behavior $X^{\prime}, Y$, or $\Sigma$, his or her best response is the one attributed to neural network pioneer Warren McCulloch: "Don't bite my finger but look where I am pointing."

\section{Rule Formation}

How does a human or monkey make sense out of a complex environment? Some sequences of actions or stimuli lead to reward or some other desired result, and other actions or stimuli do not. Hence, the organism has to generalize, if possible, from a few examples and develop a rule for which combinations of actions or stimuli are rewarding.

Human and monkey brains are not "symbolic processors" but are networks of functionally significant brain regions. High-level concepts, such as "move to the right," "green plastic," or "the number 4," correlate with brain activity in many locations. Moreover, environmental activation of these concepts, however they are represented, is continuous rather than all-or-none. At this stage of modeling, we simply assign network nodes to significant high-level concepts, we do not specify where exactly the nodes are located (though we sometimes have rough hypotheses about general regions), how many neurons each consists of (generally, we do not identify them with single neurons), or how the nodes for those concepts arose. But we note that Fuster, Bauer, and Jervey (1982) found evidence in single cell recordings from the prefrontal cortex that partially supports the idea that cell groups develop that correspond to specific classes of stimuli. These researchers showed that in one behavioral task (delayed matching to sample), during the course of the experiment particular cell groups in the prefrontal cortex learned to be selectively activated by significant stimuli or rewards related to task performance.

Before modeling rule formation, we need to break up the processes into smaller cognitive subprocesses, some of which have been modeled already. Before this, we must introduce some of the components likely to be used in these high-level network models.

\section{Network Principles}

Since neural network modeling has become more popular for psychologists, neuroscientists, and technological researchers, the field sometimes gives the appearance of being disordered and lacking in foundational principles. In reality, however, a few well-defined principles have emerged and been repeatedly used in different combinations (Levine, 1989, 1991). These principles include: (1) associative learning, to enable strengthening or weakening of connections between events based on contiguity or probable causality; (2) lateral inhibition, to enable choices between competing percepts, drives, categorizations, plans, or behaviors; (3) opponent processing, to enable selective enhancement of events that change over time; (4) neuromodulation, to enable contextual refinement of attention; (5) interlevel resonant feedback, to enable reality testing of tentative classifications; and (6) error correction (as is used in back propagation networks), to enable responses to fit as closely as possible to a predetermined plan.

These principles are not complete or independent but provide us with powerful tools for constructing models that contain biologically realistic components (even if we don't yet know that the network architectures we arrived at are exactly like those in the brain).

We can build on a large class of existing neural networks that use these principles and others in various combinations to perform cognitive tasks (e.g., Bapi \& Levine, 1994, 1995; Cohen, Dunbar, \& McClelland, 1990; Cohen \& Servan-Schreiber, 1992; Dehaene \& Changeux, 1991; Levine \& Prueitt, 1989). Let us talk about the experimental data we are modeling and then talk about some steps toward a model built on the successes of our past work.

\section{Data on Frontal Lobe Damage and Rule Disruption}

In experiments by Brody and Pribram (1978), macaque monkeys with or without prefrontal damage were taught to perform various sequences of motor behaviors (pressing particular panels) to get a food reward. One group each of frontally lesioned and normal monkeys learned that they had to press panels in a particular, invariant order (the externally ordered task). Another group each of frontal and normal monkeys could press all the panels in any order, as long as they pressed no panel twice; that is, if we denote individual movements by $A, B$, and $\mathrm{C}$, then $\mathrm{ABC}, \mathrm{ACB}, \mathrm{BAC}, \mathrm{BCA}, \mathrm{CAB}$, and CBA were all rewarded (the internally ordered task).

The results were that frontal monkeys performed as well as the normal monkeys did on the externally ordered task but had extreme difficulty with both learning and retention of the internally ordered task. In addition, Brody and Pribram (1978) found that frontal monkeys could overcome their difficulty on internal ordering if they were "sophisticated" (i.e., had previous experience with the externally ordered task). In this case, their strategy was to simplify their task by limiting their options and treating it as an externally ordered task.

Brody and Pribram (1978) concluded that intact frontal lobes are not necessary for the primary learning of motor sequences, which may involve connections between the limbic system and basal ganglia. But intact frontal lobes are apparently necessary for classification of sequencesthat is, for discerning a complex rule for which sequences are rewarded and which are not.

Bapi and Levine $(1994,1995)$ simulate these data using the network of Figure 1. In this network, one module (avalanche) learns sequences, and another module (ARTMAP) learns their connections with reward or nonreward. However, our model does not yet encode a general rule. If, say, $\mathrm{ABC}$ and $\mathrm{CBA}$ are both rewarded, the network learns to produce $A B C$ if it perceives the stimulus for $A$, and $C B A$ if it perceives the stimulus for $C$. But it cannot general- 
ize from $A B C$ and $C B A$ to the other four variations (ACB, BAC, BCA, and CAB).

This flexible sequence rule is one of many types of complex rules that require intact frontal lobes to be learned effectively. Another appears in the classical delayed alternation task (Pribram, Plotkin, Anderson, \& Leong, 1977). In this experiment, a monkey has access to two possible food trays. The location of food reward alternates between the trays on successive trials. Normal monkeys can easily learn where the reward is found. Those with frontal damage have difficulty, tending to go repeatedly to the same tray. Only if time delays are interposed, so that the lag between a reward at tray $A$ and a subsequent reward at tray $B$ is consistently longer than the lag from $\mathrm{B}$ to $\mathrm{A}$, do frontal animals reach normal performance levels.

Still another high-level rule is learned in the experiment wherein reward is always placed under the most novel object in the environment. Pribram (1961) compared normal and frontally lesioned rhesus monkeys in a scene with several junk objects. In the first stage, the monkey is presented with one cue, an object placed over 1 of 12 holes drilled in a board. A peanut has been placed under this cue. After a fixed number of trials in which the monkey lifts the cue and is thus rewarded, a second (novel) object is introduced and placed over another hole while the board is hidden from the monkey. Again, the reward (peanut) is placed under the novel cue. This process is repeated until all holes are covered. Each time the peanut remains under the novel cue until the animal finds the peanut a certain number of times. Frontally damaged animals tend to get a reward sooner, being more attracted to the novel object.

Pribram's frontal animals went earlier to the novel object than did the normal animals because the counteracting tendency to approach previously rewarded objects was weaker in frontal animals than in normal animals. But the apparent better performance of frontal monkeys is illusory since they made many random responses and did not go to the novel object reliably. After 9 or 10 objects, in fact, the normal animals caught up to and overtook the frontal animals in task performance. Levine and Prueitt (1989) conjectured that the normal monkeys switched from low-level rules, such as "the food is under the red triangle," to the high-level rule, "the food is under the novel object." The flexible sequence and delayed alternation data suggest that frontal lobes are required to learn such a high-level rule.

In summary, the prefrontal cortex seems necessary for learning any complex rule about what classes of stimuli or motor actions will lead to reward. This includes, for example, the three rules: (1) "do A, B, and C once each, in any order, with no repeats," (2) "alternate A and B," and (3) "go to the most novel object." Is there some general set of network architectures that models how the prefrontal cortex, along with motivational regions of the archicortex and limbic system and sensory and motor regions of the neocortex, extracts the regularity from the organism's experience of reward or nonreward, thereby "deciding" what the rule is for obtaining reward? Supervised neural network algorithms, such as back propagation (Rumelhart et al.,

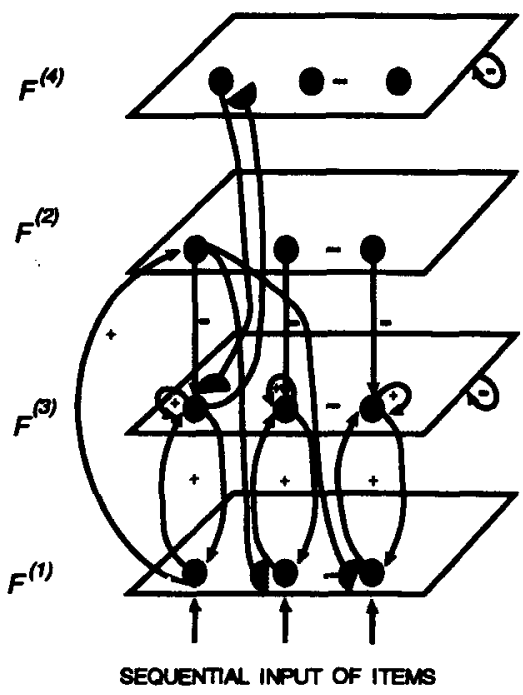

(a)

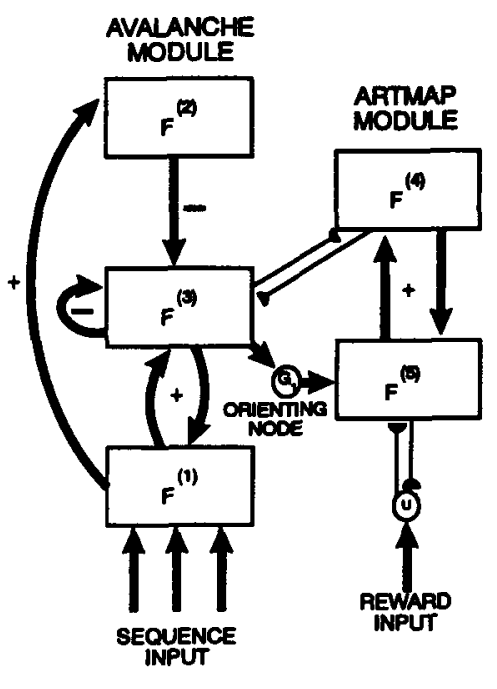

(b)

Figure 1. (a) Network for learning motor sequences. A sequence detector layer, $F^{(4)}$, is added to an avalanche module consisting of layers $F^{(1)}, F^{(2)}$, and $F^{(3)}$. (Adapted from Neural Networks, 7, R. S. Bapi \& D. S. Levine, "Modeling the Role of the Frontal Lobes in Sequential Task Performance: I. Basic Structure and Primacy Effects," p. 1173, copyright 1994, with kind permission from Elsevier Science Ltd.) (b) Network for sequence classification, based on combining the avalanche network of part (a) (with detail condensed) with a simplified version of the ARTMAP network (Carpenter et al., 1991). "*" represents excitation, "-" represents inhibition, semicircles denote modifiable connections, and arrows denote fixed weight connections. 
1986; Werbos, 1974, 1993) and ARTMAP (Carpenter et al., 1991), can learn to associate any arbitrary subset of spatial (or, by extension, spatiotemporal) pattern space with reward. However, such algorithms learn only the examples they have seen and cannot yet generalize from instances they have perceived to those they have not perceived. Without such a generalization capacity, these networks cannot yet be said to infer complex rules (see Bapi \& Levine, 1995, for discussion).

An intelligent rule learner must simultaneously consider many different levels of complexity of possible rules. Since it is not known a priori what level or type of rule will be the correct one, this involves a process that Hofstadter (1980) called a "search of search spaces." Prefrontal involvement in the search of search spaces is probably somehow related to the capacity of the prefrontal cortex to make mental images of possible alternative states (Ingvar, 1985). But what other brain areas are involved in this process? The hippocampus, since it is connected to all areas of sensory and association cortex and processes cortical inputs for memory storage, would also seem to be implicated in this high-level rule forming scheme. So would the amygdala, since it is crucial for affective evaluation of sensory stimuli.

\section{The "Trilogy" of Frontal Lobes, Amygdala, and Hippocampus}

Some suggestions for the interacting functions of these areas can be obtained from the discussion in Pribram (1991, chapters 8 and 9). Pribram reviewed a large body of literature implicating the amygdala and hippocampus in complementary roles. The amygdala functions to create a sense of familiarity, whereas the hippocampus is involved in innovation. More specifically, lesions to the amygdala interfere with processing of parts of the environment that are currently being reinforced (positively or negatively). Monkeys with amygdalar damage, for example, are less likely than are normal monkeys to avoid locations in which punishment has previously occurred. Lesions to the hippocampus, by contrast, interfere with processing of parts of the environment that are currently not being reinforced. The hippocampus is therefore necessary for processing the currently irrelevant "background," which needs to be stored in case the context should change to the point where background information becomes necessary (e.g., when the experimenter rewards an animal's approach to a previous unrewarded object). The frontal lobes are strongly connected to both limbic areas, as shown in Figure 2, and "supervise" which of the other two systems (hippocampal or amygdalar) should be engaged in a particular context.

Hence, the hippocampus is necessary for orienting to novel events. Is it too fanciful to suggest that the hippocampus is involved not only in orientation within perceptual space but also orientation within belief space? The hippocampus has feedback connections with the parts of cortex that process all sensory modalities; it deals not with raw sensory data but with storage of new associations in short-term memory, through the set of connections shown in Figure 3 (Cohen \& Eichenbaum, 1993). If rules based on the dimensions that are currently emphasized do not lead to correct predictions, I speculate that the frontal lobes (the brain's "executive") suppress their signals to the amygdala ("familiarity locus") and increase their signals to the hippocampus. The hippocampus then engages a search over perceptual dimensions (e.g., in the example of Pribram, 1961, the dimension of novelty) to which the amygdala has previously suppressed attention as irrelevant. If a rule based on one of these dimensions, which could have been encoded at any of several brain levels, turns out to predict what is reinforcing to the organism, attention shifts to that dimension. Selective attention then engages the amygdala.

\section{Conclusions?}

The networks in Figures 1-3 contain fragments of a big picture. Other fragments, not shown here for space reasons, have been falling gradually into place. For example, Parks and Levine (1995) model fronto-hippocampal interactions in episodic and semantic memory and Alzheimer dementia. The hippocampus' reorienting function leads to setting a new episode. The frontal lobes are involved in deciding which current stimuli or stimulus attributes are relevant for the current episode. Alzheimer's disease is mimicked by loss of activity in a "cholinergic" module that influences cortical and limbic areas but affects a "hippocampal" module at a faster rate than it affects a "frontal" module. The model reproduces stages of decay in performance of a semantic memory task (Verbal Fluency Test) with Alzheimer's disease, and simulations of an episodic memory task (Rey Auditory-Verbal Learning Test) are in progress. Episodic memory is impaired at an earlier stage than is semantic memory because episodic memory is affected more by hippocampal malfunction.

In another article (Levine, 1994), I propose a generic network theory of self-actualization and optimal cognition.

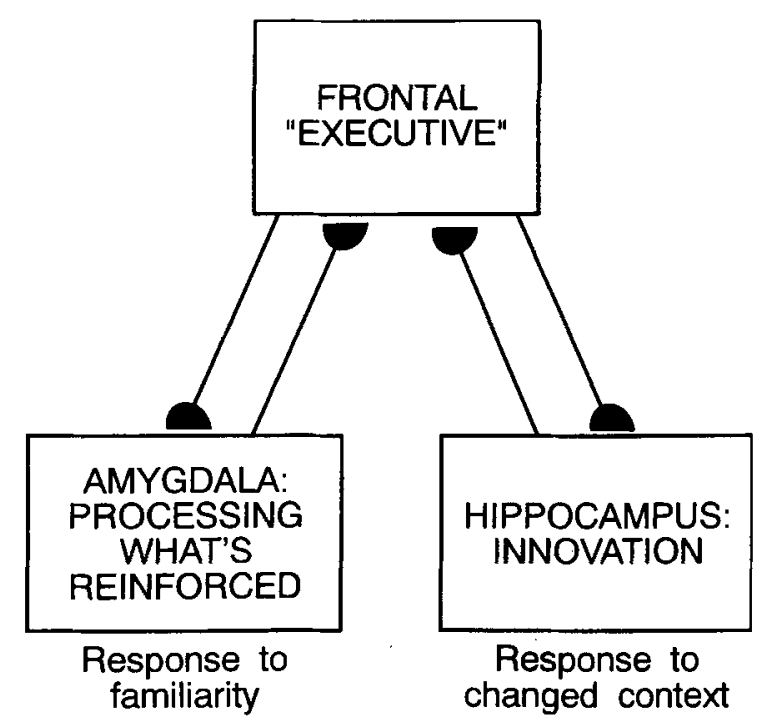

Figure 2. Schematic of three interconnected brain regions that perform different functions in rule formation. 


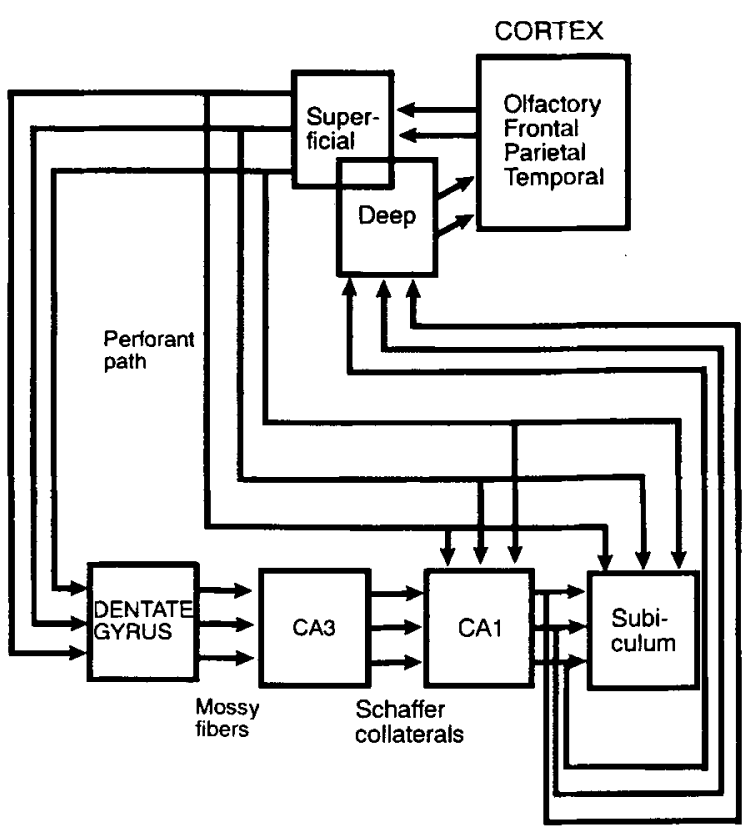

Figure 3. Schematic of main connections within the different subregions of the hippocampus. (From Memory, Amnesia, and the Hippocampal System, p. 100, by N. Cohen and H. Eichenbaum, 1993, Cambridge, MA: MIT Press. Copyright 1993 by MIT Press. Adapted with permission.)

This theory includes the frontal lobes, as modelers of alternative states of the organism's drive representations; the amygdala, as generator of a "negative affect" signal if an imagined alternative state is closer to optimal than the current state; and norepinephrine signals from the midbrain, as regulators of how many drives can be attended to. Also, there is considerable evidence about differentiated roles for subregions of the prefrontal cortex (dorsal vs. orbital) that needs to be incorporated into the network theories.

While these models are still speculative and not yet fully predictive theories, they are neural network instantiations of qualitative theories developed by psychologists and neuroscientists for behavioral and cognitive functions of specific brain areas and pathways. This is already the basis for productive partnership between computational modelers and laboratory scientists.

\section{REFERENCES}

ANDERSON, J. A., SPOEHR, K. T., \& BENNETT, D. J. (1994). A study in numerical perversity: Teaching arithmetic to a neural network. In D. S Levine \& M. Aparicio IV (Eds.), Neural networks for knowledge representation and inference (pp. 311-335). Hillsdale, NJ: Erlbaum.

APARICIO, M., IV, \& Levine, D. S. (1994). Why are neural networks relevant to higher cognitive function? In D. S. Levine \& $M$. Aparicio IV (Eds.), Neural networks for knowledge representation and inference (pp. 1-26). Hillsdale, NJ: Erlbaum.

BAPI, R. S., \& LEviNe, D. S. (1994). Modeling the role of the frontal lobes in sequential task performance: I. Basic structure and primacy effects. Neural Networks, 7, 1167-1180.

BAPI, R. S., \& LEVINE, D. S. (1995). Modeling the role of the frontal lobes in sequential task performance: II. Classification of sequences. Manuscript submitted for publication.

BREMNER, F. J., \& GotTs, S. J. (1995). Shades of Braitenberg's Vehi- cle 5. Behavior Research Methods, Instruments, \& Computers, 27, 169-172.

Brody, B. A., \& Pribram, K. H. (1978). The role of frontal and parietal cortex in cognitive processing: Tests of spatial and sequence functions. Brain, 101, 607-633.

Carpenter, G. A., Grossberg, S., \& Reynolds, J. H. (1991). ARTMAP: Supervised real-time learning and classification of nonstationary data by a self-organizing neural network. Neural Networks, 4, 565-588.

Cohen, J. D., Dunbar, K., \& McClelland, J. L. (1990). A parallel distributed processing model of the Stroop effect. Psychological Review, 97, 332-361.

Cohen, J. D., \& Servan-Schreiber, D. (1992). Context, cortex, and dopamine: A connectionist approach to behavior and biology in schizophrenia. Psychological Review, 99, 45-77.

Cohen, N. J., \& EICHENBAUM, H. (1993). Memory, amnesia, and the hippocampal system. Cambridge, MA: MIT Press.

Dehaene, S., \& Changeux, J.-P. (1991). The Wisconsin Card Sorting Test: Theoretical analysis and modeling in a neuronal network. Cerebral Cortex, 1, 62-79.

FoDOR, J. A., \& PYlyshyn, Z. W. (1988). Connectionism and cognitive architecture: a critical analysis. In S. Pinker \& J. Mehler (Eds.), Connections and symbols (pp. 3-71). Cambridge, MA: MIT Press.

Fuster, J. M., Bauer, R. H., \& Jervey, J. P. (1982). Cellular discharge in the dorsolateral prefrontal cortex of the monkey during cognitive tasks. Experimental Neurology, 77, 679-694.

Hofstadter, D. R. (1980). Gödel, Escher, Bach: An eternal golden braid. New York: Random House.

HOPFIELD, J. J., \& TANK, D. W. (1986). Computing with neural circuits: A model. Science, 233, 625-633.

INGVAR, D. H. (1985). Memory of the future. Human Neurobiology, 4, 125-136.

KOHONEN, T. (1988). Self-organization and associative memory. Berlin: Springer-Verlag.

LEVINE, D. S. (1989). Neural network principles for theoretical psychology. Behavior Research Methods, Instruments, \& Computers, 21, 213-224.

LEVINE, D. S. (1991). Introduction to neural and cognitive modeling. Hillsdale, NJ: Erlbaum.

LEVINE, D. S. (1994). Steps toward a neural theory of self-actualization. World Congress on Neural Networks, San Diego (Vol. 1, pp. 215220). Hillsdale, NJ: Erlbaum.

Levine, D. S., \& PrueitT, P. S. (1989). Modeling some effects of frontal lobe damage: novelty and perseveration. Neural Networks, 2 , 103-116.

PARKs, R. W., \& LEVINE, D. S. (1995). Neural network modeling of verbal fluency in Alzheimer's disease. Manuscript submitted for publication.

Pribram, K. H. (1961). A further experimental analysis of the behavioral deficit that follows injury to the primate frontal cortex. Journal of Experimental Neurology, 3, 432-466.

PRIBRAM, K. H. (1991). Brain and perception: Holonomy and structure in figural processing. Hillsdale, NJ: Erlbaum.

Pribram, K. H. (ED.) (1994). Origins: Brain and self organization. Hillsdale, NJ: Erlbaum.

Pribram, K. H., Plotkin, H. C., Anderson, R. M., \& Leong, D. (1977). Information sources in the delayed alternation task for normal and "frontal" monkeys. Neuropsychologia, 15, 329-340.

Rumelhart, D. E., Hinton, G. E., \& Williams, R. J. (1986). Learning internal representations by error propagation. In D. E. Rumelhart, J. L. McClelland, \& the PDP Research Group (Eds.), Parallel distributed processing: Explorations in the microstructure of cognition (Vol. 1, pp. 318-362). Cambridge, MA: MIT Press.

WERBOS, P. J. (1974). Beyond regression: New tools for prediction and analysis in the behavioral sciences. Unpublished doctoral dissertation, Harvard University.

WeRBOS, P. J. (1993). The roots of backpropagation: From ordered derivatives to neural networks and political forecasting. New York: Wiley.

(Manuscript received November 21, 1994; revision accepted for publication January 31,1995 .) 